\title{
AVALIAÇÃO DA ADIÇÃO DE DIFERENTES CONCENTRAÇÕES DE SEMENTES DE CHIA E LINHAÇA NAS CARACTERÍSTICAS FÍSICO-QUÍMICAS DO QUEIJO TIPO MINAS FRESCAL
}

\author{
D. COSTA CURTA ${ }^{1}$ e E. RIGO ${ }^{1}$ \\ ${ }^{1}$ Universidade do Estado de Santa Catarina -Departamento de Engenharia de Alimentos e \\ Engenharia Química-DEAQ \\ E-mail para contato: elisandra.rigo@udesc.br
}

\begin{abstract}
RESUMO - O desenvolvimento de formulações alimentícias é uma necessidade constante para o mercado visando otimizar processamentos e agregar diferentes ingredientes. Neste contexto, o queijo tipo minas frescal um dos mais consumidos no país, torna-se um produto alvo a ser investigado. Assim, o presente trabalho tem como objetivo avaliar as características físicoquímicas, bem como a oxidação lipídica e a concentração de minerais em queijo tipo minas frescal elaborado com a adição de diferentes concentrações de chia e linhaça. Os dados foram avaliados de acordo com o planejamento experimental onde se observou valores próximos de proteína, umidade, cinzas e $\mathrm{pH}$ para as amostras acrescidas de sementes e a formulação padrão. Para o teor de potássio e ferro, as formulações contendo sementes indicaram maior teor desses minerais devido à quantidade destes nas sementes, em relação à amostra padrão, o que ressalta a importância do produto proposto. Os teores de lipídios e oxidação lipídica apresentaram variações entre as formulações acrescidas de sementes e em relação a formulação padrão, esta foi maior, o que indica que as formulações propostas tornam-se interessantes em relação a preservação das características primordiais para produtos alimentícios em especial devido as características de acidez do queijo e traços de oxidação lipídica.
\end{abstract}

Palavras-Chave: Queijo tipo minas frescal, sementes de chia, sementes de linhaça.

DOI: $10.5965 / 24473650312017012$

\section{INTRODUÇÃO}

Dentre os mais diversos tipos de queijos, o queijo tipo Minas frescal tem ampla aceitação no mercado e faz parte do hábito da população brasileira, sendo o terceiro queijo mais consumido no País (ABIQ, 2016). Assim sendo, o melhoramento da qualidade e formulação deste, representa uma oportunidade de agregar valor nutricional ao alimento (GOMES, et al., 2011).

Vislumbrando uma alimentação mais saudável os consumidores têm procurado produtos com melhor qualidade nutricional, ou seja, produtos ricos em minerais, vitaminas, fibras, proteínas, baixo teores de sal, redução de gordura, entre outros (MORAES; COLLO, 2006). Assim, surgem os alimentos funcionais, definidos como quaisquer alimentos ou aditivos alimentares que trazem benefícios à saúde, além de possuírem nutrientes essenciais (HALSTED, 2003). 
Nesse sentido, a semente de chia (Salvia Hispanica L.) consiste em uma fonte potencial de nutrientes, devido seu aporte de proteínas, fibras, minerais e compostos fenólicos (COELHO; SALAS-MELLADO, 2014), podendo ser incluída na classe dos alimentos funcionais (TANAMATI, et al., 2012). Dentre estes, encontra-se também a linhaça (Linumusitatissimum L.), uma semente com várias aplicações, como a adição em pães, bolos, biscoitos entre outros (GALVÂO et al., 2008), considerando que em sua composição estão presentes altas taxas de fibras solúveis, vitaminas e minerais como o zinco, ferro, magnésio, potássio, cálcio e fósforo (COSKUNER; KARABABA, 2007).

Desta forma, o presente trabalho tem como objetivo avaliar as características físicoquímicas (lipídios, proteínas, umidade, cinzas), bem como a concentração de ferro e potássio, o teor de oxidação e o pH em queijo tipo minas frescal adicionado de diferentes concentrações de sementes de chia e linhaça.

\section{MATERIAL E MÉTODOS}

\subsection{Elaboração dos queijos}

Para a realização dos experimentos foram elaborados queijos, (todos com uma quantidade de 2 litros de leite de vaca) de acordo com a metodologia proposta por Monteiro, et al., (2011), sendo um deles a formulação padrão sem adição de sementes e os demais com diferentes concentrações de sementes de chia e linhaça, apresentadas na Tabela 1, definidas conforme o planejamento experimental fatorial completo $2^{2}$.

Tabela 1- Varáveis e níveis utilizados no planejamento fatorial completo $2^{2}$ para elaboração de queijo minas frescal adicionado de sementes de chia e linhaça

\begin{tabular}{cccc}
\hline & \multicolumn{3}{c}{ Níveis } \\
\cline { 2 - 4 } Variáveis independentes & -1 & 0 & 1 \\
\hline Chia (\%) & 2 & 2,5 & 3 \\
\hline Linhaça (\%) & 1 & 2 & 3 \\
\hline
\end{tabular}

Ao leite aquecido a $37^{\circ} \mathrm{C} \pm 1^{\circ} \mathrm{C}$ foram adicionados $0,04 \mathrm{~g}$ de fermento láctico composto pelas bactérias (microrganismos) Lactococcus lactis e Lactococcus cremoris; $0,8 \mathrm{ml}$ de cloreto de cálcio e 1,8ml de coalho (Há-la). Após o período da coagulação (aproximadamente 50 minutos) efetuou-se o corte da massa em cubos; a mexedura durante 20 minutos com intervalos de 5 minutos para descansar a massa; dessoragem prévia; adição das sementes segundo as concentrações definidas na Tabela 1, seguida da salga; enformagem e armazenamento por 12 horas sob-refrigeração $\left(5^{\circ} \mathrm{C} \pm 1^{\circ} \mathrm{C}\right)$. Os queijos foram embalados em embalagem Cryovac, selados a vácuo e mantidos sob-refrigeração até a realização das análises. Amostras dos queijos foram avaliadas no dia $1^{\circ}$ e no $15^{\circ}$ dia de armazenamento somente para oxidação e $\mathrm{pH}$, as demais, umidade, proteínas, lipídios, cinzas, e minerais ferro e potássio, após a elaboração, sendo para todas as analise, previamente trituradas e homogeneizadas. 


\subsection{Análises físico-químicas, de ferro e potássio e de oxidação}

As análises físico-químicas (lipídios, proteína, umidade e cinzas) realizadas nos queijos tipo minas frescal com adição de sementes de chia e linhaça foram realizadas seguindo a Instrução Normativa $\mathrm{N}^{\circ} 68$ (BRASIL, 2006). O pH foi realizado segundo metodologia definida pelo Instituto Adolfo Lutz (2008).

A concentração dos minerais, ferro e potássio foi determinada por espectrometria de absorção atômica em chama (Varian Spectra AA-55), segundo metodologia descrita por (AOAC, 1995). Os elementos foram quantiicados em condições de operação otimizada em chama ar/acetileno e acetileno/óxido nitroso, com pressão ajustada dos gases, no modo absorção. Os cálculos dos teores de ferro e potássio nas amostras foram baseados em uma curva de calibração obtida com as soluções padrões segundo a metodologia da (AOAC, 1995).

Para análise do grau de oxidação foi utilizado o Teste do Ácido 2-Tiobarbitúrico (TBA), sendo a leitura em espectrofotômetro a $531 \mathrm{~nm}$. Os resultados foram expressos em mg malonaldeído/kg amostra.

\subsection{Análise estatística}

As análises estatísticas foram desenvolvidas no software STATISTICA® 12. As médias dos dados dos resultados das análises foram aplicadas em Análises de Variância Simples (ANOVA) para verificar a influência das variáveis independentes no produto com nível de significância de $\mathrm{p} \leq 0,05 \%$.

\section{RESULTADOS E DISCUSSÃO}

\subsection{Caracterização físico-química e determinação de ferro e potássio}

Os resultados (Tabela 2) indicam que os queijos adicionados de sementes possuem teor de lipídios variados, o ponto central $(5,6$ e 7$)$ foi o que apresentou o maior valor médio $(8,27 \%)$, enquanto que as formulações 1 e 3 os menores teores $(4,84$ e $4,41 \%)$ respectivamente. A diferença entre as formulações pode ter ocorrido devido às sementes não serem trituradas, o óleo contido não ser extraído uniforme para o meio. Ainda, segundo Rowney et al. (2004) relatam que em queijos pode ocorrer a emulsificação da caseína e, subsequentemente, afetar a microestrutura dos glóbulos de gordura, devido a variação de pressão osmótica criada pelos diferentes teores de solutos adicionados a massa, ou seja, diferença nos teores de gordura pode ocorrer devido as diferentes pressões osmóticas criadas pela mistura do sal, considerando que no presente estudo a salga foi realizada após a dessoragem, esta pode não ter ocorrido de forma homogênea.

Valores de gordura encontrados por Gomes et al. (2011) para o queijo Minas frescal elaborado com leite bovino, foram entre $22,4 \%$ e $21,2 \%$, maiores que do presente estudo, possivelmente devido a variabilidade dos teores de gorduras oriundos da matéria prima, considerando a raça, fase de lactação entre outros fatores (OLIVEIRA et al., 2010). 
Tabela 2 -Matriz do planejamento fatorial completo $2^{2}$ com os valores codificados e (reais) e os valores de lipídios, proteína, umidade, cinzas, potássio e ferro em relação ao teor de sementes adicionadas ao queijo minas no tempo inicial (após o processamento)

\begin{tabular}{ccccccccc}
\hline Ensaios & \multicolumn{3}{c}{$\begin{array}{c}\text { Variáveis } \\
\text { Independentes }\end{array}$} & \multicolumn{2}{c}{ Variáveis Dependentes } \\
\hline & $\begin{array}{c}\text { Chia } \\
(\%)\end{array}$ & $\begin{array}{c}\text { Linhaça } \\
(\%)\end{array}$ & $\begin{array}{c}\text { Lipídio } \\
(\%)\end{array}$ & $\begin{array}{c}\text { Proteína } \\
(\%)\end{array}$ & $\begin{array}{c}\text { Umidade } \\
(\%)\end{array}$ & $\begin{array}{c}\text { Cinzas } \\
(\%)\end{array}$ & $\begin{array}{c}\text { Potássio } \\
(\mathrm{mg} / 100 \mathrm{~g})\end{array}$ & $\begin{array}{c}\text { Ferro } \\
(\mathrm{mg} / 100 \mathrm{~g})\end{array}$ \\
\hline 1 & $-1(2)$ & $-1(1)$ & $4,84 \pm 0,12$ & $15,98 \pm 0,71$ & $64,67 \pm 0,71$ & $2,91 \pm 0,04$ & $178,86 \pm 0,11$ & $178,86 \pm 0,11$ \\
\hline 2 & $1(3)$ & $-1(1)$ & $6,00 \pm 1,05$ & $15,67 \pm 0,58$ & $66,73 \pm 0,08$ & $2,95 \pm 0,05$ & $161,90 \pm 0,21$ & $161,90 \pm 0,21$ \\
\hline 3 & $-1(2)$ & $1(3)$ & $4,41 \pm 1,69$ & $15,41 \pm 0,03$ & $66,32 \pm 1,28$ & $3,01 \pm 0,11$ & $153,22 \pm 0,32$ & $153,22 \pm 0,32$ \\
\hline 4 & $1(3)$ & $1(3)$ & $6,01 \pm 2,17$ & $14,36 \pm 0,03$ & $65,28 \pm 0,31$ & $3,20 \pm 0,07$ & $199,42 \pm 0,11$ & $199,42 \pm 0,11$ \\
\hline 5 & $0(2,5)$ & $0(2)$ & $8,03 \pm 1,35$ & $14,45 \pm 0,06$ & $65,45 \pm 1,68$ & $2,73 \pm 0,13$ & $194,07 \pm 0,15$ & $194,07 \pm 0,15$ \\
\hline 6 & $0(2,5)$ & $0(2)$ & $8,74 \pm 1,35$ & $15,55 \pm 0,15$ & $64,61 \pm 0,10$ & $2,87 \pm 0,03$ & $185,32 \pm 0,12$ & $185,32 \pm 0,12$ \\
\hline 7 & $0(2,5)$ & $0(2)$ & $8,04 \pm 0,99$ & $15,50 \pm 0,32$ & $64,96 \pm 0,04$ & $3,07 \pm 0,02$ & $189,75 \pm 0,11$ & $189,75 \pm 0,11$ \\
\hline Padrão & - & - & $13,06 \pm 1,68$ & $16,70 \pm 0,20$ & $65,34 \pm 0,56$ & $2,85 \pm 0,01$ & $144,37 \pm 0,16$ & $144,37 \pm 0,16$ \\
\hline
\end{tabular}

Os efeitos estimados segundo planejamento fatorial completo $2^{2}$ para teor de lipídios indicou que somente a concentração de chia quadrático apresentou efeitos significativos negativo com valor de $p \geq 0,05$. Em comparação com o queijo minas frescal padrão, o teor de lipídios das formulações avaliadas no planejamento (Tabela 2) foram menores, possivelmente no processo de dessoragem, mistura do sal e sementes pode ter ocorrido uma variação da perda de gordura.

A adição das sementes não resultou em efeitos significativos, com valor de $p \geq 0,05$ no teor de proteína das formulações. O ponto central apresentou um valor de proteína de 15,17\%, enquanto as formulações 1, 2 e 3 os maiores valores de teor de proteína (15,98\%). O queijo 4 foi o que apresentou o menor valor (14,36\%). A legislação não determina valores de proteínas em queijo minas frescal, podendo variar sua composição entre 12 a 18\% (Machado, et al., 2004, Brazaca, et al., 2009). As sementes de chia e linhaça contêm quantidade moderada de proteínas, entre 12 e $20 \%$. Porém, possivelmente devido às sementes não serem trituradas, o processo de análise não foi sensível para quantificação, já que em comparação ao queijo minas frescal padrão, as formulações apresentaram teores menores.

Para o teor de umidade os queijos 1, 2 e 3 foram os que apresentaram os maiores valores $(66,73 \%)$; enquanto que os queijos da formulação central apresentaram valores menores $(65,07 \%)$. O queijo minas frescal padrão apresentou um valor de umidade de 65,34\%, próximo aos valores obtidos nas formulações com adição de sementes (Tabela 2). Os efeitos estimados quanto ao teor de umidade das formulações de queijo minas adicionado de sementes indicou que somente a concentração de chia quadrática apresentou efeitos significativos positivo com valor de $\mathrm{p} \geq 0,05$.

A formulação do ponto central apresentou valores de teor de cinzas de $2,89 \%$. Os queijos 3 e 4 foram os que apresentaram maior concentração de cinzas $(3,11 \%)$; enquanto que os queijos 1 e 20 menores valores (2,93\%). Para o queijo minas frescal padrão o teor de cinzas foi de $2,85 \%$ (Tabela 1). Observou-se que a adição da chia e linhaça não resultaram em efeitos significativos no teor de cinzas, com valor de $p \geq 0,05$. Isso se deve possivelmente a salga do queijo representar uma grande proporção das cinzas do produto (ABREU et al., 2011), visto que a quantidade de sal utilizada foi proporcional ao peso do queijo analisado, considerando que as cinzas no queijo são caracterizadas pela presença das substâncias salinas e minerais presentes no leite ou adicionadas durante a fabricação do queijo (PEREIRA et al., 2001apud 
ABREU, et al., 2011) e neste caso também do conteúdo de minerais das sementes, evidenciado pelo queijo minas frescal padrão que apresentou menor valor em comparação as formulações com semente.

No planejamento experimental para o teor de potássio, o queijo da formulação 4 apresentou o maior valor $(199,42 \mathrm{mg} / 100 \mathrm{~g})$, enquanto que a 3 o menor valor $(153,2 \mathrm{mg} / 100 \mathrm{~g}$, conforme podemos observar na Tabela 2. Marques, (2008) indica que a quantidade de potássio presente no grão de linhaça é de $869 \mathrm{~g} / 100 \mathrm{~g}$ outra literatura $813 \mathrm{~g} / 100 \mathrm{~g}$ (USDA, 2016). Para as sementes de chia, o potássio corresponde a $407 \mathrm{mg} / 100 \mathrm{~g}$ (Usda, 2016). Analisando os resultados obtidos (Tabela 2), verificou-se que a formulação (4) contendo $3 \%$ de sementes foi a que apresentou o maior valor de potássio, seguida pela formulação do ponto central, indicando que as formulações com maiores quantidades de sementes, principalmente a linhaça, correspondem aos maiores teores de minerais.

Para o teor de ferro, segundo o planejamento experimental, os queijos 3 , 4 e o ponto central, foram os que apresentaram os maiores valores, em torno de $0,50 \mathrm{mg} / 100 \mathrm{~g}$, enquanto que os queijos 1 e 2 apresentaram os menores valores $(0,34$ e $0,39 \mathrm{mg} / 100 \mathrm{~g})$ respectivamente. Marques, (2008) indica que a quantidade de ferro presente no grão de linhaça é de 4,7 mg/100g outra literatura, 5,7 mg/100g (USDA, 2016). Para as sementes de chia, o ferro corresponde a 7,7 mg/100g (Usda, 2016). Analisando os resultados obtidos (Tabela 2), verificou-se que a formulação (4) contendo $3 \%$ de sementes foi a que apresentou o maior valor de ferro, seguida pela formulação do ponto central. Considerando a quantidade de sementes utilizadas nas formulações, verifica-se que quanto maior a quantidade principalmente de chia, maior será o teor de ferro presente nos queijos, devido ela possuir maior quantidade deste mineral. Para o queijo minas frescal padrão, a concentração de potássio e ferro foi inferior as formulações acrescidas de semente, o que era esperado, ressaltando que o queijo tipo minas frescal padrão não contém sementes.

\subsection{Oxidação e valores de pH}

A Tabela 3 mostra os resultados obtidos no $1^{\circ}$ dia de fabricação e após $15^{\circ}$ de armazenamento. Os valores de $\mathrm{pH}$ das formulações no primeiro dia após a fabricação variaram de 6,05 (valor médio do ponto central), a 5,92 para formulação 3 e de 6,44, 6,49 e 6,45 para 1, 2 e 4 respectivamente. Para o queijo padrão o valor foi de 6,50. Resultados próximos foram encontrados por Gomes et al. (2011), em queijo Minas frescal produzidos com leite de vaca, em torno de 6,2. Após 15 dias da sua elaboração, os resultados indicaram que os queijos com menor concentração de sementes de linhaça apresentaram valores de $\mathrm{pH}$ maior em relação ao $1^{\circ}$ dia (formulações 1 e 2). A formulação padrão variou de 6,50 para 6,69 . 
Tabela 3 - Matriz do planejamento fatorial completo $2^{2}$ com os valores codificados e (reais) e os valores de oxidação (Ox) e pH em relação ao teor de sementes adicionadas ao queijo minas após a fabricação e ao completar 15 dias de armazenamento

\begin{tabular}{ccccccc}
\hline \multirow{2}{*}{ Formulações } & \multicolumn{2}{c}{ Variáveis } \\
\cline { 2 - 7 } & $\begin{array}{c}\text { Chia } \\
(\%)\end{array}$ & $\begin{array}{c}\text { Linhaça } \\
(\%)\end{array}$ & $\begin{array}{c}\text { Ox Inicial } \\
(\mathrm{mg} \text { mal./Kg } \\
\text { amostra })\end{array}$ & $\begin{array}{c}\text { Ox15 dias } \\
(\mathrm{mg} \text { mal./Kg } \\
\text { amostra) }\end{array}$ & $\begin{array}{c}\mathrm{pH} \\
\text { Inicial }\end{array}$ & $\begin{array}{c}\mathrm{pH} \\
15 \text { dias }\end{array}$ \\
\hline 1 & $-1(2)$ & $-1(1)$ & $0,138 \pm 0,22$ & $0,401 \pm 0,22$ & 6,44 & 6,84 \\
\hline 2 & $1(3)$ & $-1(1)$ & $0,112 \pm 0,12$ & $0,434 \pm 0,12$ & 6,49 & 6,84 \\
\hline 3 & $-1(2)$ & $1(3)$ & $0,001 \pm 0,01$ & $0,203 \pm 0,01$ & 5,92 & 6,11 \\
\hline 4 & $1(3)$ & $1(3)$ & $0,115 \pm 0,12$ & $0,288 \pm 0,12$ & 6,45 & 6,59 \\
\hline 5 & $0(2,5)$ & $0(2)$ & $0,070 \pm 0,11$ & $0,281 \pm 0,11$ & 5,81 & 5,81 \\
\hline 6 & $0(2,5)$ & $0(2)$ & $0,010 \pm 0,17$ & $0,258 \pm 0,17$ & 6,1 & 6,02 \\
\hline 7 & $0(2,5)$ & $0(2)$ & $0,027 \pm 0,33$ & $0,220 \pm 0,33$ & 6,25 & 6,12 \\
\hline Padrão & 0 & 0 & $0,358 \pm 0,13$ & $0,606 \pm 0,13$ & 6,50 & 6,69 \\
\hline
\end{tabular}

A alta umidade presente nos queijos é normalmente o principal motivo pela elevação dos resultados de $\mathrm{pH}$ e consequentemente diminuição da sua conservação (PRUDÊNCIO, et al., 2006). Já o ponto central apresentou uma diminuição de $\mathrm{pH}$ com valor médio inicial de 6,05 para 5,98 após 15 dias de estocagem. Buriti, Rocha e Saad (2005) estudaram o queijo Minas frescal por 21 dias armazenado à temperatura de $8{ }^{\circ} \mathrm{C}$ e observaram uma redução no $\mathrm{pH}$ de 6,16 a 5,38 (diferença de 0,78 ) entre o $1^{\circ}$ dia e o $21^{\circ}$ dia de armazenamento.

De acordo com o planejamento experimental as amostras de queijos 1, 2 e 4 apresentaram valores acima que $0,100 \mathrm{mg}$ manolaldeído/ $\mathrm{kg}$ amostra; enquanto que a formulação do ponto central e a amostra 3 apresentaram valores menores que $0,100 \mathrm{mg}$ manolaldeído/kg amostra. Para o queijo minas frescal padrão o resultado obtido foi de $0,358 \mathrm{mg}$ manolaldeído/kg amostra. Cabe ressaltar que quando o teor de MA (Manomaldeído) é muito baixo, possivelmente outros aldeídos não provenientes do processo de degradação dos lipídios podem reagir com o (Ácido 2-Tiobarbitúrico) TBA (FRANKEL, 1993; JADHAV et al., 1996; BERSET; CUVELIER, 1996; SAIDIA; HAMMOND, 1989, SILVA, et al.,1999).

Com relação ao grau de oxidação 15 dias após a sua elaboração, os resultados indicaram pequena variação em relação ao tempo inicial, sendo os resultados obtidos abaixo ou próximo a 0,400 mg manolaldeído/kg amostra .Em relação ao queijo minas frescal padrão, verificou-se aumento de $0,248 \mathrm{mg}$ manolaldeído/ $\mathrm{kg}$ em relação ao valor inicial de $0,358 \mathrm{mg}$ manolaldeído/kg. Contudo cabe ressaltar que as formulações com menores quantidades de sementes e maior teor de umidade foram as que apresentaram os maiores aumentos nos valores de oxidação.

\section{CONCLUSÃO}

O estudo realizado indicou que a adição de sementes de chia e linhaça aos queijos minas frescal proporcionou uma melhor qualidade nutricional, a concentração de lipídios reduziu em relação ao queijo minas frescal padrão. Com relação à concentração de potássio e ferro, a formulação (4) adicionada de 3\% sementes apresentou maior valor, o que indica que à medida 
que aumenta a concentração de sementes, verifica-se também a elevação do conteúdo destes minerais no produto. A oxidação teve relação somente com a umidade.

\section{REFERÊNCIAS BIBLIOGRÁFICAS}

ABREU, L. R. et al..Caracterização física e química de queijos parmesão ralado comercializados na região sul de minas gerais. Rev. Inst. Latic. Cândido Tostes, Mar/Abr, v.66, n.379, p.16-24, 2011.

AOAC. Association of Official Analytical Chemists. Official methods of analysis of the Association of the Analytical Chemists, 16th ed., AOAC International: Washington, 1995.

ASSOCIAÇÃO BRASILEIRA DAS INDÚSTRIAS DE QUEIJO- ABIQ. Queijos no Brasil. 2016. Disponível em: 〈http://www.abiq.com.br/nutricao_queijosbrasil_tipos_vaca.asp>. Acesso em: 31 Ago. 2016.

BERSET, C.; CUVELIER, M.-E.; Sciences des Aliments. 1996, 16, 219.

BRASIL. Ministério da Agricultura, Pecuária e Abastecimento. Métodos analíticos oficiais físico-químicos para controle de leite e produtos lácteos. Instrução Normativa $n^{\circ} 68$, de 12 de Dezembro de 2006.

BRAZACA, S. G. C., et al.. Estudo da vida útil de queijo minas. Ciência e Tecnologia de Alimentos, Campinas, v.29, n.2, p.262-269, abr.-jun. 2009.

BURITI, F. C. A.; ROCHA, J. S.; SAAD, S. M. I. Incorporation of Lactobacillus acidophilus in Minas fresh cheese and implicatons for textural and sensorial properties during storage. International Dairy Journal, v.15, n.12, p.1279-1288, Dez. 2005.

COELHO, M. S.; SALAS-MELlADO, M. de Las M.. Revisão: Composição química, propriedades funcionais e aplicações tecnológicas da semente de chia (Salviahispanica L) em alimentos. Campinas, v. 17, n. 4, p. 259-268, out./dez. 2014. Disponível em: < http://www.scielo.br/pdf/bjft/v17n4/1981-6723-bjft-17-4-259.pdf> Acesso em: 01 set. 2016.

COSKUNER, Y.; KARABABA, E. Some physical properties of flaxseed (LinumusitatissimumL.). J. Food Eng. - Elsevier, v. 78, n. 3, p. 1067-1073, 2007.

FRANKEL, E. N.; Trends Food Sci. \& Technol. 1993, 4, 220.

GALVÃO, E. L. et al. Avaliação do potencial antioxidante e extração subcrítica do óleo de linhaça. Centro de Ciência e Tecnologia de Alimentos, Campinas, jul.-Set. 2008. Disponível em: <http://www.scielo.br/pdf/cta/v28n3/a08v28n3.pdf>. Acesso em: 13 jun. 2013. 
GOMES, A. P. et al. Manufacture of low-sodium Minas fresh cheese: Effect of the partial replacement of sodium chloride with potassium chloride. Journal of Dairy Science. p. 2701-2706, 2011.

HALSTED, C. H. Dietary supplements and functional foods: 2 sides of a coin?.American Journal of Clinical Nutrition, v.77, p.1001-1007, 2003.

INSTITUTO ADOLFO LUTZ. Métodos físico-químicos para análise de alimentos, 4 ed., 1 ed. digital. São Paulo: Instituto Adolfo Lutz, 2008. 1020 p.

JADHAV, S. J. et al. Food Antioxidants:Technological, Toxicological, and Health Perspectives; Inc.; New York 1996; p. 5.

MACHADO, E. C. et al. Características físico-químicas e sensoriais do queijo Minas artesanal produzido na região do Serro, Minas Gerais. Ciência e Tecnologia de Alimentos, v. 24, n. 4, p. 516-521, out./dez. 2004.

MARQUES, Anne y C..Propriedades funcionais da linhaça (LinumUsitatissimum L.) em diferentes condições de preparo e uso em alimentos. Universidade Federal de Santa Maria. Dissertação de mestrado: Santa Maria, 2008. Disponível em: $<$ http://cascavel.cpd.ufsm.br/tede/tde_busca/arquivo.php?codArquivo=2363>. Acesso em: 26 Nov. 2013.

MONTEIRO, A. A., et al. Tecnologia de produção de derivados do leite. 2. Ed. Viçosa (MG): Ed. UFV, 2011.

MORAES, F. P; COLLO, L. M. Alimentos funcionais e nutracêuticos: Definições, legislação e benefícios à saúde. Revista Eletrônica de Farmácia, v. 3. p. 109-122, 2006. Disponível em: <https://revistas.ufg.br/REF/article/viewFile/2082/2024> Acesso em: 01 set. 2016.

OLIVEIRA, E. N. A.,et al. Composição físico-química de leites em diferentes fases de lactação. Rev. Acad., Ciênc. Agrár. Ambient., Curitiba, v. 8, n. 4, p. 409-415, 2010.

PRUDÊNCIO, E. et al. Avaliação Tecnológica e Físico-Química de Queijo Tipo Minas Frescal Obtido a Partir do Leite Concentrado por Ultrafiltração. Universidade Federal de Santa Catarina, Florianópolis, 2006. Disponível em: <http://pgeal.ufsc.br/files/2011/01/Avalia\%C3\%A7\%C3\%A3o-Tecnol\%C3\%B3gica-eF\%C3\%ADsico-Qu\%C3\%ADmica-de-Queijo-Tipo-Minas-Frescal-Obtido-a-Partir-doLeite-Con.pdf> Acesso em: 01 set. 2016.

ROWNEY, M. et al. Salt-induced structural changes in 1-day old Mozzarella cheese and the impact upon free oil formation. International Dairy Journal, p. 809-816, 2004.

SAIDIA, B.; HAMMOND, E. G.; J. Am. Oil Chem. Soc. 1989, 66, 1097. 
SILVA, F. A. M., et al. Métodos para avaliação do grau de oxidação lipídica e da capacidade antioxidante. Química Nova, 22(1) (1999). Disponível em: <http://scielo.br/pdf/qn/v22n1/1143.pdf.> Acesso em: 05 Nov. 2013.

TANAMATI, A. et al..Avaliação do teor de lipídios totais e da composição em ácidos graxos de sementes Salvia Hispânica L (Chia). XVII Seminário de iniciação cientifica e tecnológica da UTFPR, 2012. Disponível em: <http://conferencias.utfpr.edu.br/ocs/index.php/sicite/2012/paper/viewFile/390/389>. Acessoem: 13 jun. 2013.

United States Departament of agriculture USDA. National Nutrient Database for Standard Reference Release 28 slightly revised May, 2016. Disponível em:

$<$ https://ndb.nal.usda.gov/ndb/foods/show/3610?fg=\&manu=\&lfacet=\&format=\&count $=\& \max =35 \&$ offset $=\&$ sort $=\& q$ lookup=12006> . Acesso em: 26 Nov. 2013.

\section{TERMO DE RESPONSABILIDADE}

Declaro, para todos os fins de direito e que se fizerem necessários, que o conteúdo e as ideias expressas nesse artigo periódico são de total responsabilidade dos autores. 